\title{
c-Kit and stem cell factor regulate PANC-1 cell differentiation into insulin- and glucagon-producing cells
}

\author{
Yuexiu $\mathrm{Wu}^{1,2}$, Jinming $\mathrm{Li}^{1,2}$, Saira Saleem ${ }^{1,2}$, Siu-Pok Yee ${ }^{3, *}$, Anandwardhan A Hardikar ${ }^{4}$ and Rennian Wang ${ }^{1,2,5}$
}

Recent evidence has shown that stem cell factor (SCF) and its receptor, c-Kit, have an important role in pancreatic islet development by promoting islet cell differentiation and proliferation. In this study, we examined the role of c-Kit and SCF in the differentiation and proliferation of insulin- and glucagon-producing cells using a human pancreatic duct cell line (PANC-1). Our study showed that increased expression of endocrine cell markers (such as insulin and glucagon) and transcription factors (such as PDX-1 and PAX-6) coincided with a decrease in CK19 ${ }^{+}$and c-Kit ${ }^{+}$cells $(P<0.001)$ during PANC-1 cell differentiation, determined by immunofluorescence and qRT-PCR. Cells cultured with exogenous SCF showed an increase in insulin ${ }^{+}(26 \%)$ and glucagon ${ }^{+}(35 \%)$ cell differentiation $(P<0.01)$, an increase in cell proliferation $(P<0.05)$ and a decrease in cell apoptosis $(P<0.01)$. siRNA knockdown of $c$-Kit resulted in a decrease in endocrine cell differentiation with a reduction in PDX-1 and insulin mRNA, as well as the number of cells immunostaining for PDX-1 and insulin. Taken together, these results show that c-Kit/SCF interactions are involved in mediating islet-like cluster formation and islet-like cell differentiation in a human pancreatic duct cell line.

Laboratory Investigation (2010) 90, 1373-1384; doi:10.1038/labinvest.2010.106; published online 7 June 2010

KEYWORDS: PANC-1 cells; c-Kit receptor tyrosine kinase; stem cell factor; islet cell differentiation; transcription factor

Islet transplantation is a promising method for the cure of diabetes. ${ }^{1}$ However, the limited availability of cadaveric donors greatly hampers the application of this method., ${ }^{2,3}$ Therefore, multiple alternative methods are being explored to generate insulin-producing cells in vitro. ${ }^{4,5}$ A better understanding of the factors responsible for islet cell growth and differentiation is important to develop an unlimited supply of islets.

c-Kit is a receptor tyrosine kinase that binds stem cell factor (SCF). It has been well established that c-Kit/SCF interaction is critical for the survival and development of stem cells involved in hematopoiesis, pigmentation and reproduction. ${ }^{6}$ Recently, studies in the human and rat fetal pancreas have shown that c-Kit is expressed in islets and its ligand SCF is present in the pancreatic mesenchyme. ${ }^{7-9}$ Oberg-Welsh and Welsh ${ }^{7}$ reported that c-Kit/SCF interaction promoted an increase in insulin content, indicating its role in the maturation of pancreatic $\beta$-cells. We recently examined the c-Kit expression pattern in the developing rat $^{10}$ and human fetal pancreas, ${ }^{9,11}$ as well as in epithelial monolayers derived from postnatal rat islets. ${ }^{12}$ Our studies showed pronounced changes in the distribution and population dynamics of c-Kit-expressing cells during islet development and differentiation, suggesting that $\mathrm{c}$-Kit may have an important role. ${ }^{9,10} \mathrm{c}-\mathrm{Kit}^{+}$epithelial monolayers derived from postnatal rat islets can give rise to new $\beta$-cells that secrete insulin in a glucose-responsive manner. ${ }^{12}$ Furthermore, a study of $\mathrm{c}-\mathrm{Kit}^{\mathrm{W}-\mathrm{v}}$ mutant mice indicated that a lack of functional c-Kit receptors affected $\beta$-cell mass and disrupted $\beta$-cell maturation and function. ${ }^{13}$ Taken together, these reports suggest that c-Kit and its ligand, SCF, are important partners in mediating islet cell differentiation and function.

PANC-1 is a cell line that was clonally reported to be derived from human pancreatic duct carcinoma and expresses a high level of c-Kit. ${ }^{14,15}$ It can be induced to differentiate into hormone-producing islet-like clusters ${ }^{16}$ following stimulation by the FGF2 growth factor. ${ }^{16}$ To further understand the role of c-Kit/SCF in islet cell differentiation, we used exogenous SCF treatment and siRNA knockdown of $c$-Kit in PANC-1 cells to analyze the functional roles of

\footnotetext{
${ }^{1}$ Children's Health Research Institute, University of Western Ontario, London, ON, Canada; '²Department of Physiology and Pharmacology, University of Western Ontario, London, ON, Canada; ${ }^{3}$ Departments of Oncology and Biochemistry, University of Western Ontario, London, ON, Canada; ${ }^{4}$ Diabetes and Pancreas Biology Group, O'Brien Institute, Melbourne, Victoria, Australia and ${ }^{5}$ Department of Medicine, University of Western Ontario, London, ON, Canada

Correspondence: Dr R Wang, Victoria Research Laboratories, Room A5-140, 800 Commissioners Road East, London, ON, Canada N6C 2V5. E-mail: rwang@uwo.ca

${ }^{*}$ Current address: Department of Genetics and Developmental Biology, University of Connecticut Health Center. 
c-Kit/SCF in islet cell differentiation. Our results showed that $\mathrm{c}$-Kit/SCF interaction significantly promotes PANC-1 cell differentiation into hormone-expressing endocrine cells with increased cell proliferation and decreased apoptosis, providing further evidence of the importance of $\mathrm{c}$-Kit/SCF in islet development.

\section{MATERIALS AND METHODS PANC-1 Cells and Cell Culture}

PANC-1 cells (American Type Culture Collection, Manassas, VA, USA) were cultured in Dulbecco's modified Eagle's medium (Invitrogen, Burlington, ON, Canada) containing $10 \%$ fetal bovine serum (FBS) with a medium change every other day. Cells were subcultured when they reached near confluence.

\section{Differentiation culture of PANC-1 cells}

To induce PANC-1 cell differentiation, ${ }^{16}$ cells were harvested by $0.05 \%$ trypsin (Invitrogen) to prepare single cell suspension, seeded in 12-well plates and cultured in a modified differentiation medium (DME/F12 medium (Sigma, St Louis, MI, USA) containing $1 \%$ BSA, transferrin (Sigma, $5.5 \mu \mathrm{g} / \mathrm{ml}$ ) and insulin-like growth factor $1(10 \mathrm{ng} / \mathrm{ml}$, ID Labs, London, ON, Canada) for a period of 7 days. The medium was changed every other day, using gravity to allow sediment of clusters, and single or dead cells and debris were removed in the suspension. PANC-1 cells before differentiation stimulation (day $0(\mathrm{~d} 0)$ ) and islet-like clusters differentiated from PANC- 1 cells at $\mathrm{d} 3$ and $\mathrm{d} 7$ of the culture were collected and processed for immunofluorescent staining, western blot analyses and qRT-PCR assays.

\section{Differentiation culture with SCF treatment}

An initial dose-dependent (20,50, 100 and $200 \mathrm{ng} / \mathrm{ml})$ study of recombinant human (rh)SCF (ID Labs) was conducted to determine the effects of SCF on PANC-1 cell differentiation. Cells cultured with vehicle (10 $\mathrm{mM}$ acetic acid) was used as a control, $50 \mathrm{ng} / \mathrm{ml} \mathrm{rhSCF}$ was shown to be the optimal dose. Cells were cultured for 7 days and harvested for immunofluorescent staining and qRT-PCR assays. To investigate whether c-Kit/SCF interaction could affect the differentiation of other c-Kit ${ }^{+}$cell lines, we also tested the human embryonic kidney 293 cell line (HEK293 cells) ${ }^{17}$ and the rat pancreatic acinar-cell line (AR42J cells) ${ }^{18-20}$ (Supplementary Figures 1-5) using the same culture conditions described above.

\section{Differentiation culture with c-Kit( $h$ ) siRNA transfection}

To determine the effect of $\mathrm{c}-\mathrm{Kit} / \mathrm{SCF}$ interaction on PANC-1 cell differentiation, PANC-1 cells were transfected in the differentiation medium for $30 \mathrm{~h}$ with c-Kit(h) siRNA (sc29225) or control siRNA (sc-36869, proprietary sequence) using an siRNA transfection kit (Santa Cruz Biotechnology, Santa Cruz, CA, USA) with at least four different cultures per experimental group. ${ }^{9}$ Seventy-two hours after transfection, exogenous rhSCF at a final concentration of $50 \mathrm{ng} / \mathrm{ml}$ was added to the islet-like cluster cultures until d7 of differentiation when the clusters were harvested and assessed for the expression of PDX-1 and insulin at both mRNA and protein levels.

\section{Immunofluorescence and TUNEL Assay}

Cells harvested from each experimental group were fixed in $4 \%$ paraformaldehyde and embedded in $2 \%$ agarose for processing in paraffin blocks. ${ }^{21}$ Sections of $4-\mu \mathrm{m}$ thickness were cut and stained with appropriate dilutions of the primary antibodies as listed in Supplementary Table 1. Fluorescent secondary antibodies were obtained from Jackson Immunoresearch Laboratories (West Grove, PA, USA) and 4'-6-diamidino-2-phenylindole (DAPI, Sigma) was used for nuclear counterstaining. Cell proliferation was examined using Ki67 labeling (Supplementary Table 1). To identify the coexpression of phenotypes, double immunofluorescent staining for insulin, glucagon, CK19 or c-Kit was performed. Endogenous alkaline phosphatase expression in PANC-1 cells was examined by staining freshly harvested cells with Naphtol AS-MX phosphate and Fast Blue (Sigma). ${ }^{12}$ The percentage of positive cells from each experimental group and time point was determined by counting a minimum of 500 cells per sample from at least 3 different cultures. Data were expressed as a percentage of positive cells relative to the total number of cells counted.

The terminal deoxynucleotidyl transferase-mediated nick end labeling (TUNEL) assay was performed on the experimental groups. Briefly, cell sections were deparaffinized and pretreated with $0.1 \%$ trypsin, then incubated with the TUNEL reaction mixture conjugated with fluorescein-dUTP (Roche, Montreal, QC, Canada). ${ }^{21}$ The nuclei were counterstained by DAPI.

\section{RNA Isolation and Real-Time RT-PCR}

Total RNA was isolated from PANC-1 cells and PANC-1differentiated islet-like clusters using the RNAqueous-4PCR kit (Ambion, Austin, TX, USA). ${ }^{11}$ cDNA was synthesized using SuperScriptTM II reverse transcriptase (Invitrogen). The primers used are listed in Supplementary Table 2. Realtime PCR analyses were performed in a Chromo4 real-time PCR unit (Bio-Rad Laboratories, Mississauga, ON, Canada). Data were normalized to the $18 \mathrm{~S}$ rRNA subunit, with four independent repeats per experimental group and time point. ${ }^{11}$ Relative gene expression was calculated based on the $2^{\Delta \Delta C_{\mathrm{T}}}$ method as PCR signals from the $\mathrm{d} 3$ - or $\mathrm{d} 7$ differentiated PANC-1 islet-like clusters relative to PANC-1 cells $(\mathrm{d} 0){ }^{9}$

\section{Western Blot Analysis}

PANC-1 cells were collected and lysed in a Nonidet-P40 lysis buffer. In all, $20 \mathrm{~g}$ of cell lysate proteins were separated by SDS-PAGE (7.5\% for c-Kit and 12\% for SCF) and transferred to a nitrocellulose membrane (Bio-Rad Laboratories) as described previously. ${ }^{9}$ Membranes were incubated with 
polyclonal rabbit anti-c-Kit and goat polyclonal anti-SCF (Santa Cruz Biotechnology) antibodies, followed by probing with appropriate horseradish peroxidase-conjugated secondary antibodies. Proteins were detected using ECL-Plus western blot detection reagents (Perkin-Elmer, Wellesley, MA, USA) and exposed to BioMax MR Film.

\section{SCF ELISA}

To examine the amount of soluble SCF present in the medium during cell differentiation, culture media were collected before differentiation ( $\mathrm{d} 0)$ and at $\mathrm{d} 3$ and $\mathrm{d} 7$ after differentiation. Soluble SCF was measured using a human SCF enzyme-linked immunosorbent assay (ELISA) kit (ALPCO, Salem, New Hampshire, USA) with a sensitivity of $10.8 \mathrm{pg} / \mathrm{ml}$, according to the manufacturer's instructions. The amount of soluble SCF in each sample medium was calculated according to an SCF standard curve; data are expressed in $\mathrm{ng} / \mathrm{ml}$.

\section{Statistical Analysis}

Data are represented as mean \pm s.e.m. Student's $t$-test was used to assess statistical difference between two groups or one-way ANOVA (analysis of variance), followed by the least significant difference group comparison test, was used for multiple experimental groups. Differences were considered to be statistically significant at $P<0.05$.

\section{RESULTS \\ Characteristics of Undifferentiated PANC-1 Cells}

In a serum-containing medium, PANC-1 cells proliferate as epithelial-like angular cells in an adherent monolayer (Figure 2a). A high number of these cells express c-Kit ( $97 \pm 1.3 \%$, $n=3$; Figure 1a and d). However, only a few cells showing SCF expression were detected ( $3 \pm 0.4 \%, n=3$; Figure $1 \mathrm{~b}$ and $\mathrm{d})$. To better understand the phenotype of PANC-1 cells before differentiation, duct, endocrine and undifferentiated cell
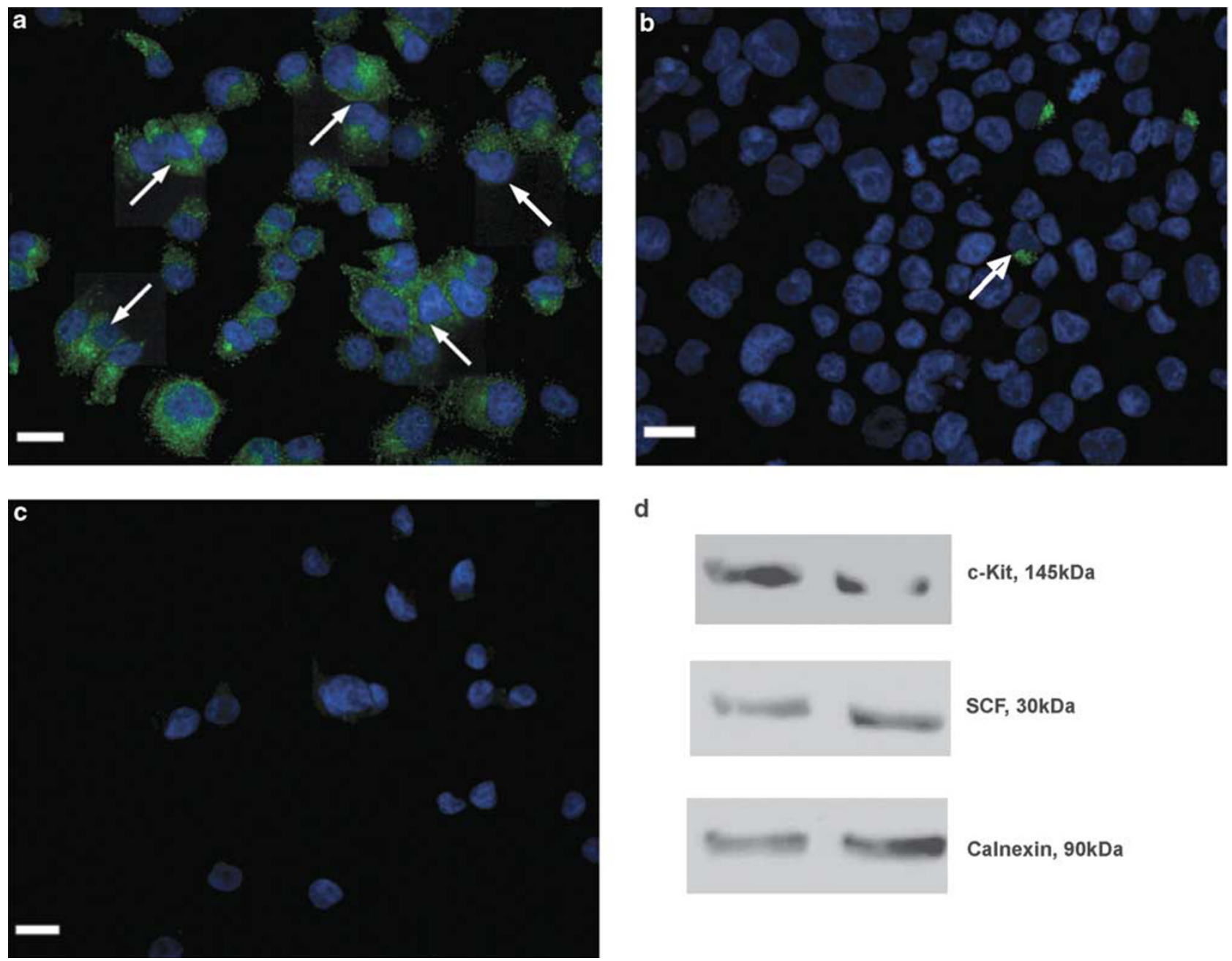

d
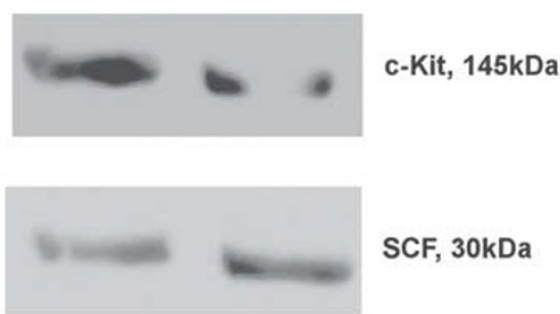

$\mathrm{SCF}, 30 \mathrm{kDa}$

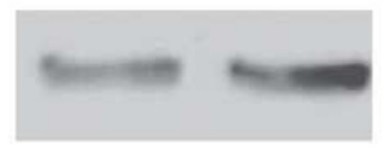

Calnexin, 90kDa

Figure 1 Expression of c-Kit and SCF in PANC-1 cells. (a-c) Representative immunostaining image of c-Kit (panel a), SCF (panel b) and c-Kit negative control (panel c) in PANC-1 cells. Positive cells were labeled by FITC (green), and the nuclei were counterstained by DAPI (blue). Arrows indicate positive cells. Scale bar: $25 \mu \mathrm{m}$. (d) Western blot analysis of C-Kit and SCF expression in two passages of PANC-1 cells. 
markers, as well as transcription factors were assessed by immunofluorescent staining. Numerous $\mathrm{CK} 19^{+} / \mathrm{Ki} 67^{+}$cells were observed (Figure $2 \mathrm{~b}$ and $\mathrm{j}$ ), indicating a proliferative duct cell phenotype. The endocrine cell-specific markers (insulin, Figure 2c; $\mathrm{C}$ peptide, Figure 2d; and glucagon, Figure $2 \mathrm{e}$ ) and the transcription factors (PDX-1, Figure 2f; PAX-6, Figure 2g)
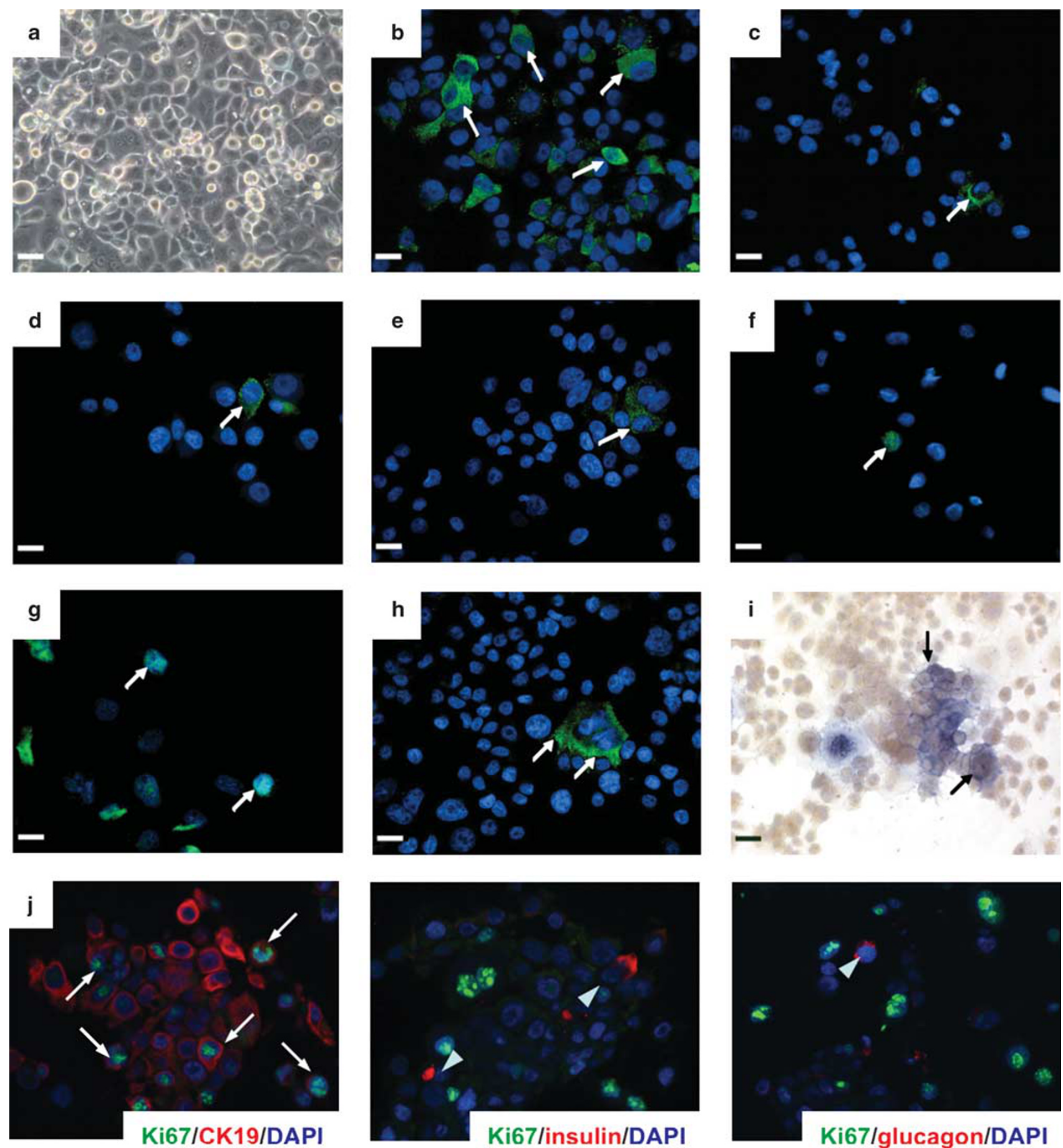

Figure 2 Characteristics of PANC-1 cell phenotypes. (a) Phase-contrast micrograph of PANC-1 cells. (b-h) Representative immunostaining images of PANC-1 cells: CK19 ${ }^{+}$(panel b), insulin ${ }^{+}$(panel c), C peptide ${ }^{+}$(panel d), glucagon ${ }^{+}$(panel e), PDX-1 ${ }^{+}$(panel f), PAX- $6^{+}$(panel g) and $\alpha \mathrm{FP}{ }^{+}$(panel h) cells were labeled with FITC (green), and the nuclei were counterstained with DAPI (blue). (i) Histochemical staining for alkaline phosphatase (blue). (j) Double immunofluorescent staining for Ki67 (green) with CK19, insulin or glucagon (red). Arrows indicate positive or double-positive cells, arrowheads indicate insulin $^{+}$or glucagon ${ }^{+}$cells that lack Ki67 labeling. For panels a and i, scale bar $=50 \mu \mathrm{m}$. For panels b-h, scale bar $=25 \mu \mathrm{m}$. 
were rarely detected $(<1-2 \%)$. These insulin ${ }^{+}$and glucagon ${ }^{+}$cells that were present did not colabel with Ki67, suggesting little to no proliferative capacity (Figure 2j). In addition, the undifferentiated cell markers, (fetoprotein $(\alpha \mathrm{FP}$, Figure $2 \mathrm{~h}$ ) and alkaline phosphatases (AKP, Figure 2i)), were also observed, but not nestin (neural precursor cell marker ${ }^{22}$ ) or OCT4 (undifferentiated embryonic stem cell marker ${ }^{23}$ ) (data not shown). These results show that PANC-1 cells cultured in the serum-containing growth medium rarely exhibit endocrine cell characteristics.

\section{Differentiated PANC-1 Islet-Like Clusters Display the Endocrine Phenotype}

When cultured in a differentiation medium, PANC-1 cells aggregated into islet-like clusters (Figure $3 \mathrm{a}$ ), similar to what has been described previously by others. ${ }^{16,24}$ There was a significant decrease in CK19 expression from d0 to d7 (Figure 3b; $P<0.001)$. In parallel to this decrease in ductal-like cells, there was an increase in the number of cells expressing endocrine cell markers (Figure 3c; $P<0.001$ ), accompanied by increased expression of PDX-1 and PAX-6 (Figure 3c; $P<0.001$ ). Results of C-peptide immunostaining (Figure $3 \mathrm{c}$ ) were consistent with those of insulin expression, indicating that newly synthesized insulin granules were present in the differentiated PANC-1 islet-like clusters. qRT-PCR analysis confirmed significantly increased levels of $P D X-1, P A X 6$, insulin and glucagon mRNA on $\mathrm{d} 3$ and $\mathrm{d} 7$ after differentiation (Figure 3d). Interestingly, the number of cells in the islet-like clusters expressing the c-Kit receptor was reduced $\sim 30 \%$ during differentiation culture (Figure $4 \mathrm{a} ; P<0.001$ ), whereas the number expressing SCF increased $\sim 75 \%$ (Figure $4 \mathrm{~b}$; $P<0.001)$. The level of soluble SCF in the medium remained unchanged as determined by ELISA (Figure 4c). To further characterize the differentiation of PANC-1 cells, cell proliferation and apoptosis were examined by immunostaining for Ki67 and the TUNEL assay respectively. The number of cells immunostained for Ki67 decreased significantly from d3 to d7 (Figure $4 \mathrm{~d} ; P<0.001$ ). In contrast, the number of cells in the islet-like clusters undergoing apoptosis increased (Figure 4e; $P<0.001$ ). Thus, differentiated PANC-1 cells display typical islet endocrine characteristics.

\section{Effects of Exogenous rhSCF Treatment on PANC-1 Cell Differentiation, Proliferation and Apoptosis}

To evaluate exogenous rhSCF treatment on PANC-1 cell differentiation, PANC-1 cells were cultured in the differentiation medium for 3 or 7 days with $50 \mathrm{ng} / \mathrm{ml}$ of rhSCF, and the expressions of PDX-1, PAX6 and endocrine cell markers were examined. There were no significant changes after 3 days of differentiation with or without rhSCF treatment (data not shown). However, after 7 days of differentiation with rhSCF treatment, immunostaining showed a significant increase in the number of insulin ${ }^{+}$and glucagon ${ }^{+}$cells, 5.5 and $6.4 \%$, respectively, as compared with controls (Figure 5a; $P<0.01$ ). The number of PDX-1- expressing cells also increased significantly $(P<0.05)$, whereas there was only a slight increase in $\mathrm{PAX}^{+}$cells (Figure 5a). qRT-PCR analysis of cultured cells showed that the mRNA levels of PDX-1, PAX6, insulin and glucagon were not significantly changed after rhSCF treatment (Figure 5a and b), which may be attributed to the large variation between the sample groups. Coexpression of insulin with CK19, c-Kit and glucagon were observed frequently after rhSCF treatment (Figure 6). Interestingly, insulin ${ }^{+}$cells were distributed in the core of many islet-like clusters, whereas glucagon ${ }^{+}$cells were present in the peripheral perimeter (Figure 6). Furthermore, analysis of cell proliferation and cell death by Ki67 and TUNEL staining, respectively, showed that the proliferative capacity of differentiated islet-like cluster cells was increased in cells cultured with rhSCF (Figure $7 \mathrm{a}$ and $b ; P<0.05)$, in parallel with a decrease in the number of cells undergoing apoptosis (Figure $7 \mathrm{a}$ and $\mathrm{c} ; P<0.05$ ). These results showed that exogenous rhSCF enhanced PANC-1 cell differentiation into islet endocrine phenotypes, increased cell proliferation and decreased apoptosis.

Interestingly, both HEK293 and AR42J c-Kit ${ }^{+}$cells also aggregated into islet-like clusters when cultured in the differentiation medium (Supplementary Figure 2). However, only AR42J cells showed a differentiation profile similar to PANC-1 cells after SCF treatment, with an increase in the number of cells immunostaining for PDX-1 and insulin and a parallel increase in PDX-1 and insulin mRNA levels (Supplementary Figure 3). HEK293 cells showed almost no endocrine cell differentiation in the clusters, with very low levels of PDX-1 and insulin mRNA and protein expression after treatment with SCF (Supplementary Figure 4), indicating that $\mathrm{c}-\mathrm{Kit} / \mathrm{SCF}$ differentiation effects differ according to cell types. However, SCF did induce cell proliferation in both AR42J and HEK293 cell lines (Supplementary Figure 5). Although no direct differentiation to pancreatic endocrine cells has been reported for HEK293 cells, AR42J cells have been shown to have the ability to differentiate into endocrine cell lineages. ${ }^{18-20}$

\section{c-Kit is Involved in PANC-1 Cell Differentiation}

To examine the effect of knockdown of c-Kit expression on PANC-1 cell differentiation, we used specific human c-Kit siRNA. $^{9}$ A significant downregulation of $\mathrm{c}$-Kit protein expression was observed $72 \mathrm{~h}$ after transfection as shown by western blot analyses $(0.58 \pm 0.04$ of $\mathrm{c}$-Kit siRNA vs $0.95 \pm 0.02$ of control siRNA; $P<0.05)$. This decrease in c-Kit expression resulted in a reduction in $P D X-1(P<0.05)$ and insulin $(P<0.01)$ mRNA levels in rhSCF-treated PANC-1 differentiation islet-like cell clusters at d7 (Figure 8a). There was a parallel decrease in the number of PDX $-1^{+}(P<0.01)$ and insulin ${ }^{+}(P<0.05)$ cells relative to controls (Figure 8b). These data provide additional evidence that $\mathrm{c}$-Kit has an important role in endocrine cell differentiation of PANC-1 cells. 

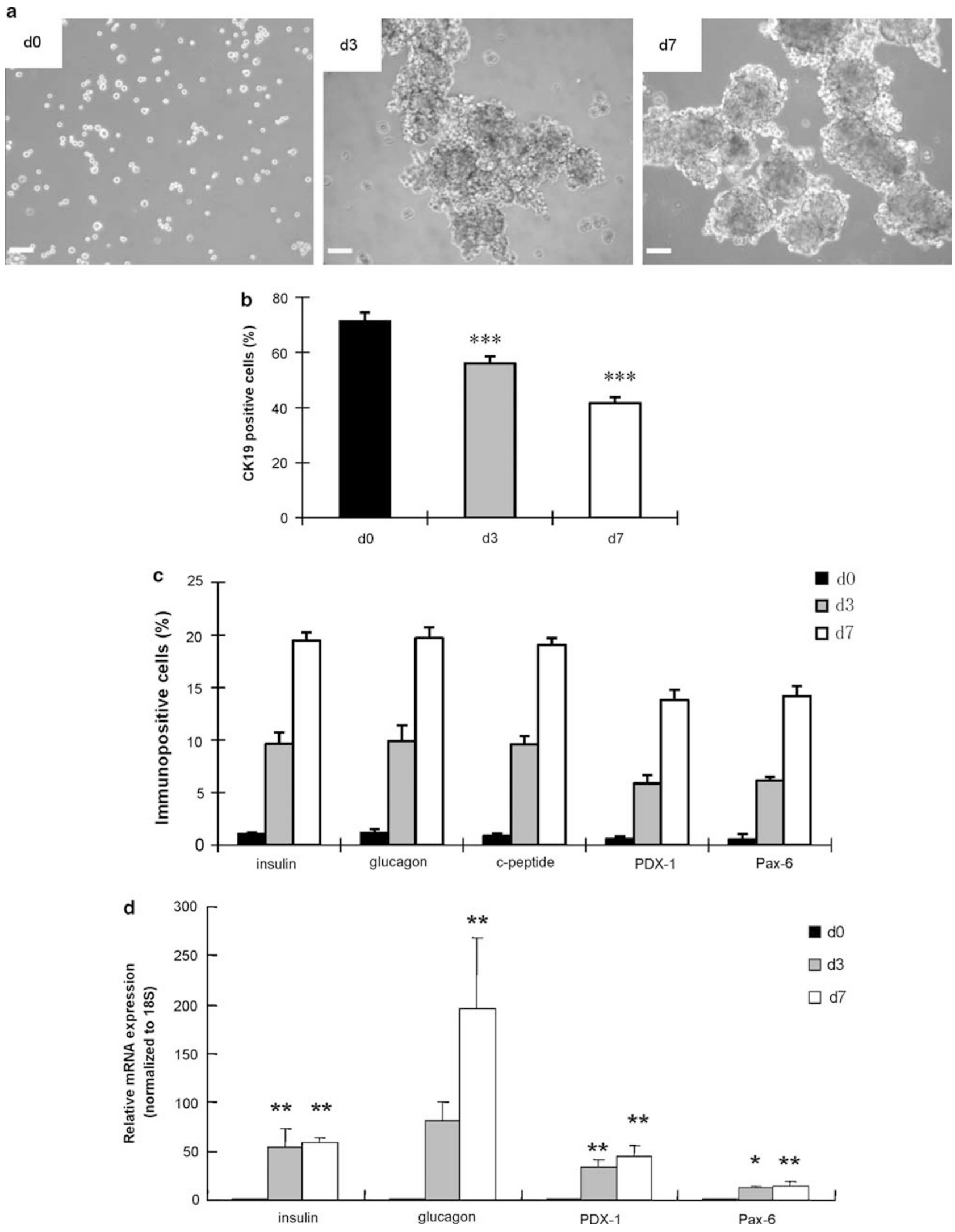


\section{DISCUSSION}

This study examines the role of c-Kit/SCF on islet cell differentiation from the PANC-1 cell line, using exogenous rhSCF treatment and siRNA knockdown of c-Kit. Exogenous SCF treatment enhanced differentiation of PANC-1 cells into islet-like cell clusters characterized by an increase in cells expressing insulin, glucagon, PDX-1 and PAX-6. Knockdown of $c$-Kit mRNA resulted in a reduction in islet cell differentiation. These results indicate that $\mathrm{c}-\mathrm{Kit} / \mathrm{SCF}$ interactions are involved in mediating PANC-1 cell differentiation into islet-like clusters.

PANC-1 is a human pancreatic cancer cell line with ductal cell characteristics when cultured in the expansion medium. ${ }^{14,15}$ However, only $70 \%$ of these cells expressed the duct cell marker CK19, suggesting a phenotypic variability. Indeed, immunofluorescent staining showed that $97 \%$ of PANC-1 cells were $\mathrm{c}-\mathrm{Kit}^{+}$, with $<1-2 \%$ of the cells expressing endocrine transcription factors (PDX-1 and PAX6) and cell markers (insulin and glucagon), whereas a small population of PANC- 1 cells were $\alpha$-fetoprotein ${ }^{+}$and alkaline phosphatase ${ }^{+}$, representing an undifferentiated cell population. ${ }^{10,12}$ Thus, the PANC-1 cell line consists of a heterogeneous cell population, with the major cell type relatively undifferentiated.

It has been shown that PANC-1 cells can be induced to differentiate into hormone-expressing islet-like clusters when cultured in serum-free differentiation medium. ${ }^{16,24,25}$ Therefore, the PANC-1 cell line has been used as a model of islet cell differentiation in several studies. ${ }^{16,24,25}$ Using our differentiation medium, we also observed that PANC-1 cells aggregated into islet-like clusters within $72 \mathrm{~h}$, and showed a significant increase in the number of cells expressing PDX-1, PAX-6 insulin and glucagon, in parallel to a significant reduction in $\mathrm{CK} 19^{+}$cells. PDX-1 is a master regulator for pancreatic development and for the maintenance of $\beta$-cell phenotypes in both humans and rodents. ${ }^{26,27}$ PAX-6 is a
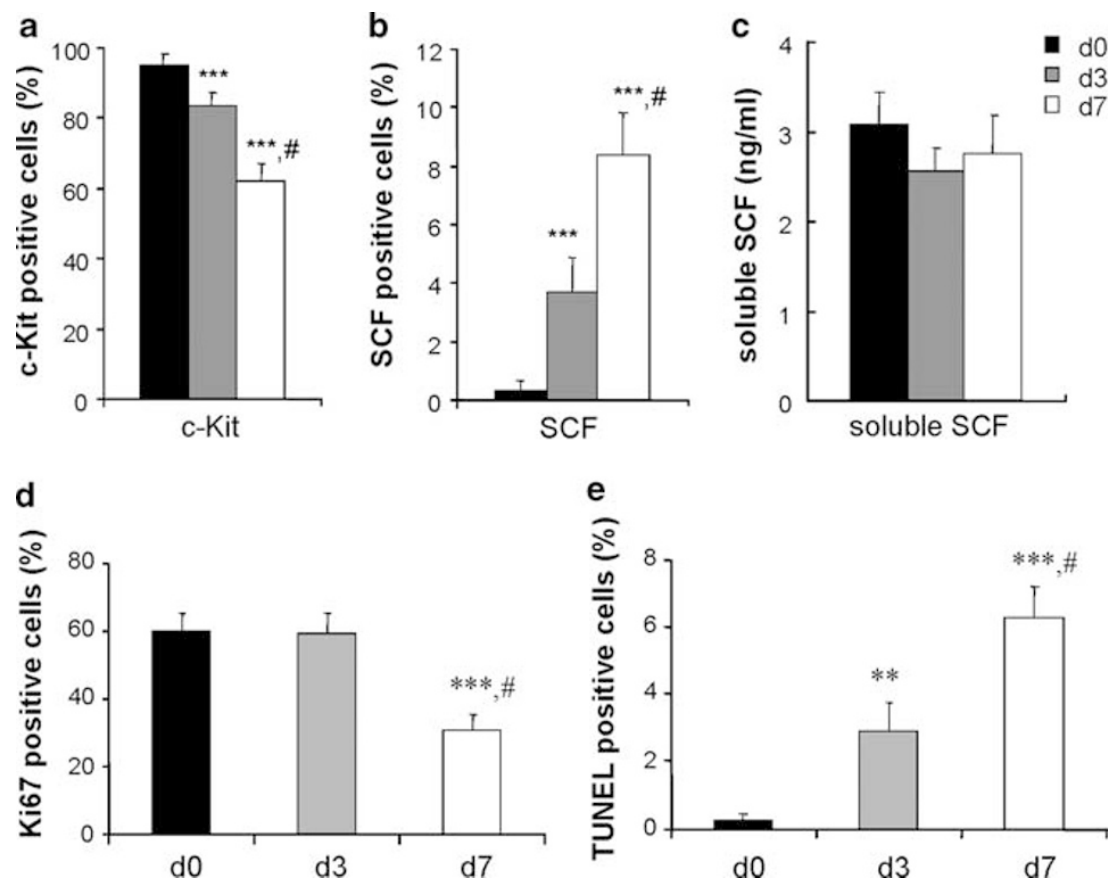

Figure 4 Expression of c-Kit, SCF, Ki67 and TUNEL during islet-like cell cluster differentiation from PANC-1 cell differentiation culture. (a, b) Quantitative analysis of c-Kit ${ }^{+}$cells (panel a) and SCF ${ }^{+}$cells (panel b) before differentiation (d0) and after 3 (d3) and 7 (d7) days of differentiation. Data are expressed as mean \pm s.e.m. ( $n=5$ experiments per group per time point; ${ }^{* * *} P<0.001$ vs d0. ${ }^{*} P<0.001$ vs d3) (c): ELISA assay for soluble SCF in the medium. Data are expressed as mean \pm s.e.m. ( $n=3$ experiments per group per time point). (d, e): Quantitative analysis of Ki67 ${ }^{+}$(panel d) and TUNEL ${ }^{+}$(panel e) cells before differentiation (d0) and after 3 (d3) and 7 (d7) days of differentiation. Data are expressed as mean \pm s.e.m. ( $n=4$ experiments per group per time point; ${ }^{* *} P<0.01,{ }^{* * *} P<0.001$ vs $\mathrm{d} 0 ;{ }^{*} P<0.001$ vs $\left.\mathrm{d} 3\right)$.

Figure 3 Characteristics of islet-like cell clusters differentiated from PANC-1 cells. (a) Phase-contrast images of PANC-1 cell aggregates during differentiation. Scale bar: $100 \mu \mathrm{m}$. (b, c) Quantitative analysis of CK19 ${ }^{+}$cells (panel b), cells expressing endocrine cell markers and transcription factors (panel c), before differentiation (d0) and after $3(\mathrm{~d} 3)$ and 7 (d7) days of differentiation. Data are expressed as mean \pm s.e.m. $(n=5$ experiments per group per time point). One-way ANOVA analysis showed significant differences between d0 and d3 or d7, as well as between d3 and d7 for all experimental groups; ${ }^{* * * P}<0.001$. (d) Real-time RT-PCR analysis of insulin, glucagon, PDX-1 and PAX- 6 mRNA expression. Data are normalized to the 18S rRNA subunit and expressed as a fold change from d0 (means \pm s.e.m., $n=4-6$ experiments per group per time point; ${ }^{\star} P<0.05,{ }^{\star *} P<0.01$ vs $\mathrm{d} 0$ ). 

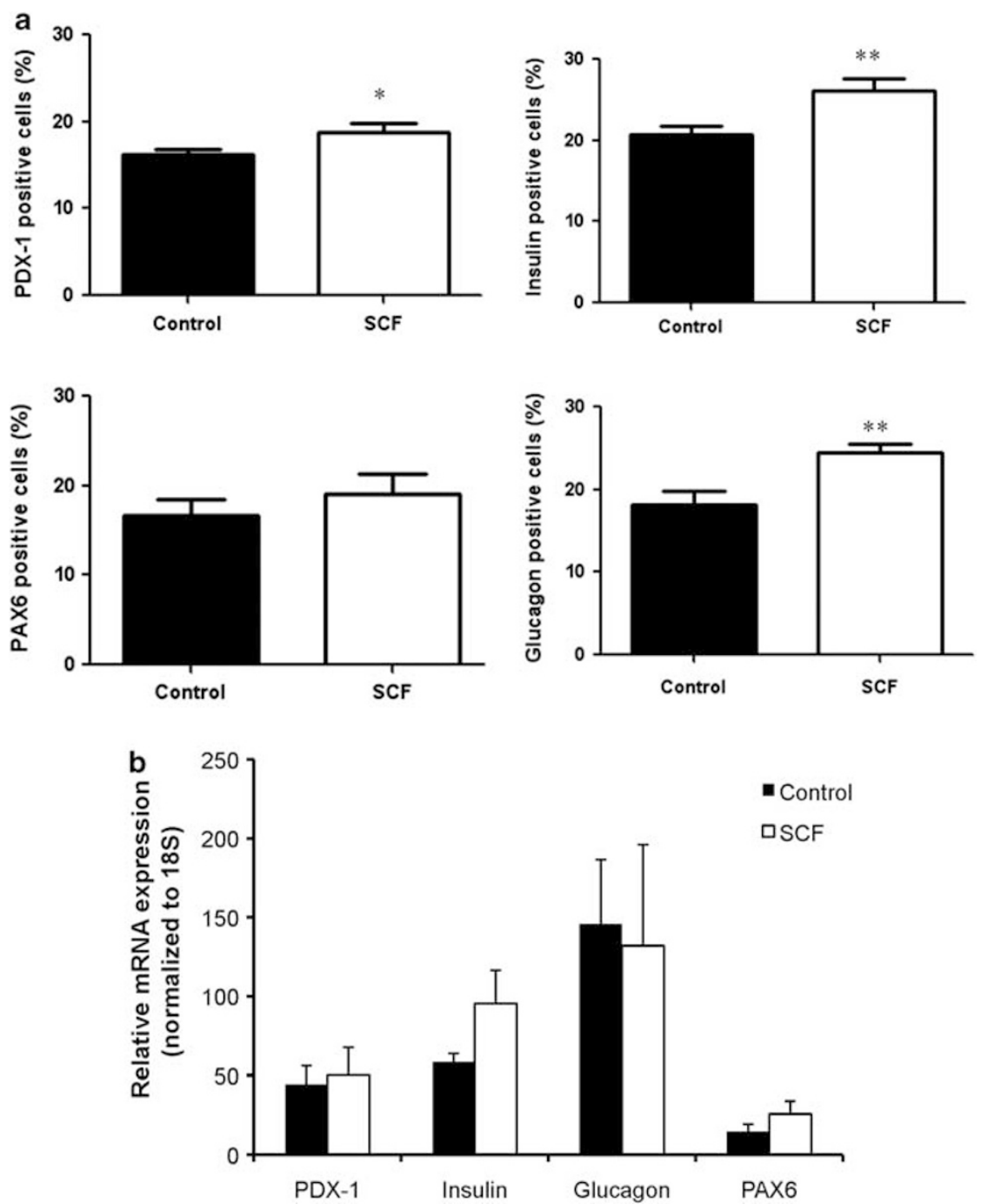

Figure 5 Exogenous rhSCF treatment enhanced differentiation of endocrine cells from PANC- 1 cells. (a) The percentage of PDX- $1^{+}$, PAX- $^{+}{ }^{,}$, insulin ${ }^{+}$and glucagon ${ }^{+}$cells in both SCF treated and control groups after 7 days of differentiation culture. Data are expressed as mean \pm s.e.m. $(n=4-8$ experiments per group; ${ }^{*} P<0.05,{ }^{*} P<0.01$ vs controls). (b) Real-time RT-PCR analysis of PDX-1, insulin, glucagon and PAX-6 mRNA expression. Data are normalized to the $18 \mathrm{~S}$ rRNA subunit and expressed as means \pm s.e.m. ( $n=4$ experiments per group).

specific transcription factor of the $\alpha$-cell lineage. ${ }^{28,29}$ Increased PDX-1 and PAX-6 expression indicates that islet cell differentiation from PANC-1 cells involved the upregulation of important transcription factors in pancreatic development. The mRNA expression levels of PDX-1 and PAX6 were lower than those of insulin and glucagon. This may be due to the fact that: (1) the peak time for expression of these transcription factors is earlier than for the endocrine cell markers, (2) there is a negative feedback effect of the increase in the number of endocrine cells or (3) other transcription factors are involved. However, only $\sim 25 \%$ of PANC-1 cells differentiated into endocrine/transcription factor + cells, which is likely due to the suboptimal conditions of stimulation. This suggests that it is necessary to develop better microenvironments in vitro to optimize islet cell differentiation from PANC-1 cells.
During differentiation, we found a significant increase in the number of cells expressing SCF and a significant decrease in the number of $\mathrm{cKit}^{+}$cells, whereas the level of soluble SCF in the medium remained consistent. This has also been reported to occur during hematopoietic stem cell differentiation into terminal erythroid or myeloid cells, as well as human erythroleukemia cell line differentiation. ${ }^{30}$ In addition, in endothelial cells, IL-1 downregulated c-Kit expression while upregulating SCF expression, suggesting that the balance of c-Kit and SCF in the vessel wall is important in inflammation. ${ }^{31}$ Decreased c-Kit expression may be due to an increase in c-Kit receptor degradation, which is triggered by increased binding of $\mathrm{SCF}^{32}$ and may indicate a mechanism to limit SCF effects during the inflammation process. These data suggest that the balance of c-Kit and SCF may also be important during PANC-1 cell differentiation into islet-like cell clusters. 

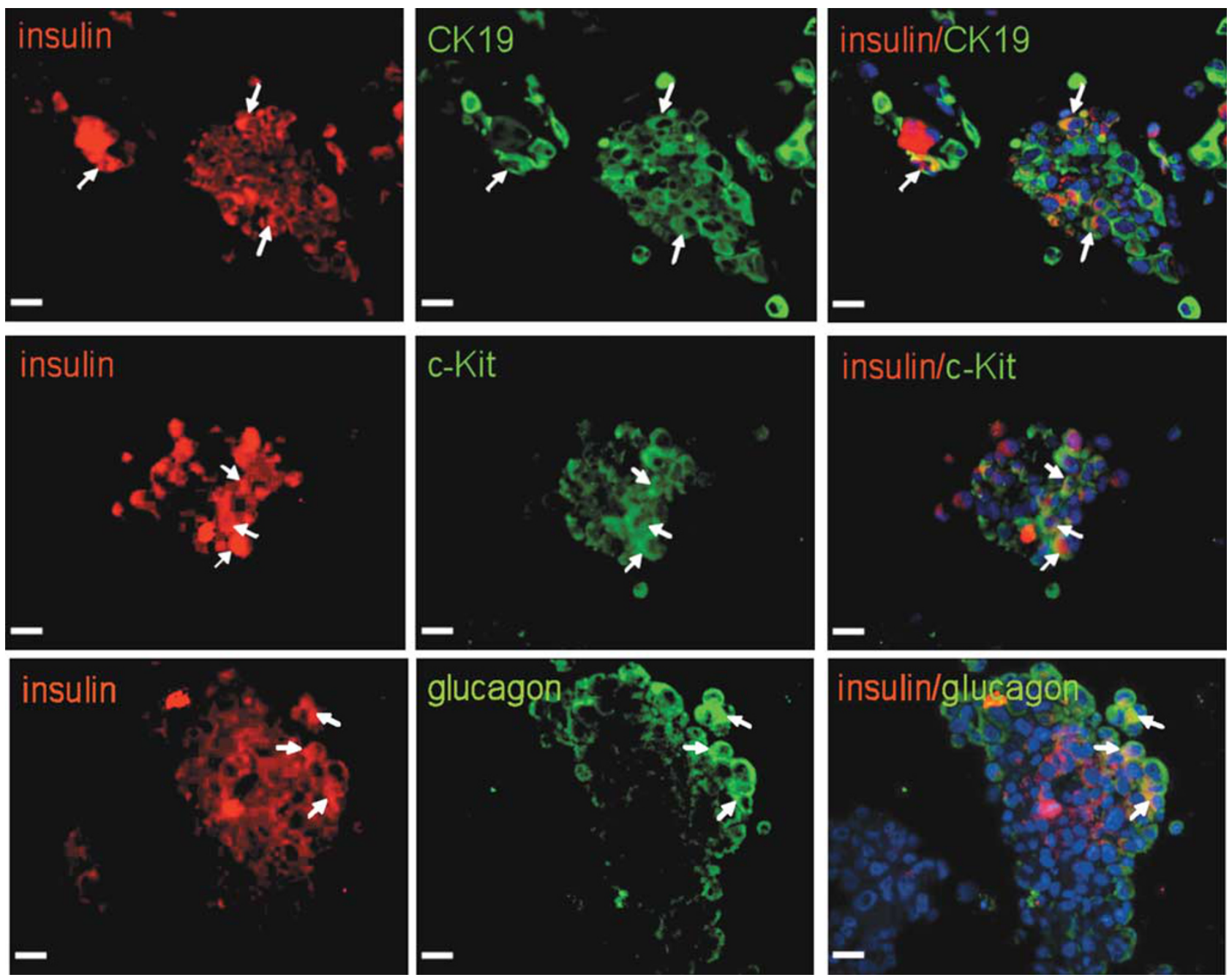

Figure 6 Double immunofluorescent staining for insulin/CK19, insulin/c-Kit and insulin/glucagon in an islet-like cell cluster 7 days after differentiation culture with SCF treatment. Representative images are shown. Insulin ${ }^{+}$cells were labeled by TRITC (red); CK19 ${ }^{+}, \mathrm{c}-K i t^{+}$and glucagon $^{+}$cells were labeled by FITC (green); and the nuclei were counterstained by DAPI (blue). Arrows indicate the costaining cells. Scale bar: $25 \mu \mathrm{m}$.

To determine whether c-Kit and SCF have a role in PANC-1 cell differentiation, we first performed an upregulation of SCF by treating cells with exogenous rhSCF. Differentiation of cultured cells was improved, as shown by an additional $5.5 \%$ increase in insulin ${ }^{+}$cells and an additional $6.4 \%$ increase in glucagon ${ }^{+}$cells. However, these increases in endocrine cell differentiation were less than we anticipated. In the normal circulation, the level of soluble SCF level is $1-3 \mathrm{ng} / \mathrm{ml} .{ }^{33}$ As the dosage we used for SCF treatment is $50 \mathrm{ng} / \mathrm{ml}$, which is much higher than the normal circulating level, this suggests that exogenous rhSCF is not as effective as endogenous SCF. Endogenous SCF exists in both a transmembrane and a soluble form. It has been reported that these two forms of SCF have different functions: ${ }^{34}$ the soluble form of SCF shows a relatively limited ability to promote primordial germcell survival compared with the transmembrane form of SCF. ${ }^{35,36}$ During PANC-1 cell differentiation, we observed a significant increase in the number of cells expressing transmembrane SCF, whereas the level of soluble SCF remained unchanged, suggesting that $\mathrm{c}$-Kit positive PANC-1 cells are also more responsive to transmembrane SCF. Thus, the different functions of the two forms of SCF may explain the relatively modest effect of exogenous SCF on PANC-1 cell differentiation.

Besides the effect of exogenous SCF on cell differentiation, we found that cell proliferation and survival rates were improved significantly in the SCF-treated group. Similar effects were observed previously in studies of hematopoietic cells $^{37-39}$ and hepatocytes. ${ }^{40}$ Furthermore, SCF prevents hematopoietic progenitor cells from apoptosis. ${ }^{31,41}$ Decreased apoptosis may contribute to the increased number of cells expressing insulin and glucagon during the differentiation of PANC-1 cells.

To identify a specific role for c-Kit in PANC-1 cell differentiation, we examined the effects of siRNA-mediated c-Kit knockdown. A $40 \%$ reduction in c-Kit protein levels in 

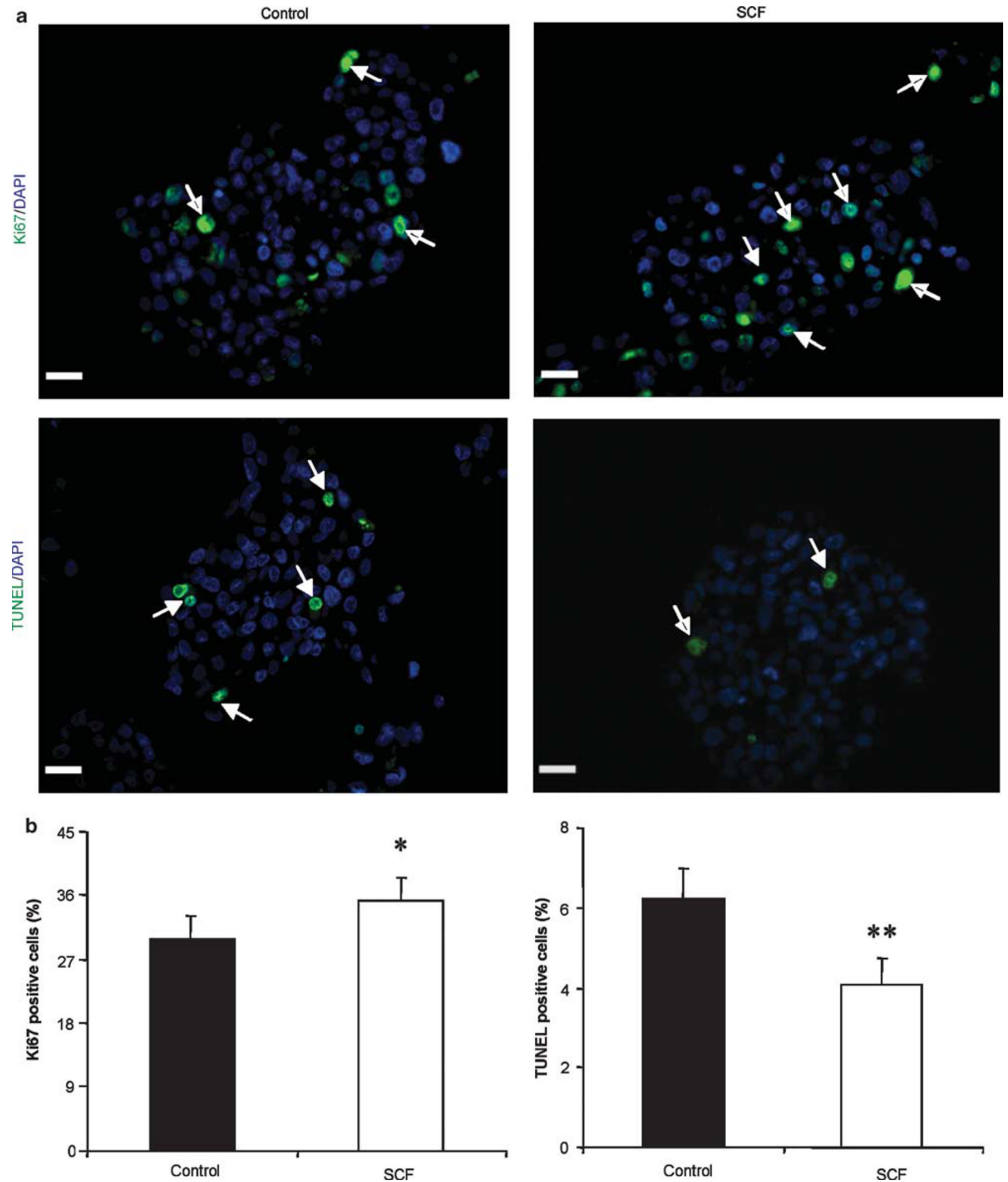

Figure 7 Effect of rhSCF on cell proliferation and apoptosis during differentiation culture. (a) Representative immunofluorescent images of Ki67 and TUNEL at day 7 of differentiation culture with or without SCF. Ki67 ${ }^{+}$and TUNEL ${ }^{+}$cells were labeled by FITC (green), and the nuclei were counterstained by DAPI (blue). Arrows indicate positive cells. Scale bar: $25 \mu \mathrm{m}$. (b, c) The percentage of Ki67 ${ }^{+}$and $\mathrm{TUNEL}^{+}$cells in the islet-like cell clusters. Data are expressed as mean \pm s.e.m. ( $n=5$ experiments per group; ${ }^{\star} P<0.05,{ }^{* *} p<0.01$ vs controls).

PANC-1 cells resulted in a significant reduction in PDX-1 and insulin mRNA levels, as well as in the number of PDX- $1^{+}$and insulin $^{+}$cells in differentiated islet-like cell clusters. These results indicate that c-Kit is involved in insulin-producing cell differentiation of PANC-1 cells and are in accord with our recent reports that activation of the c-Kit 
a

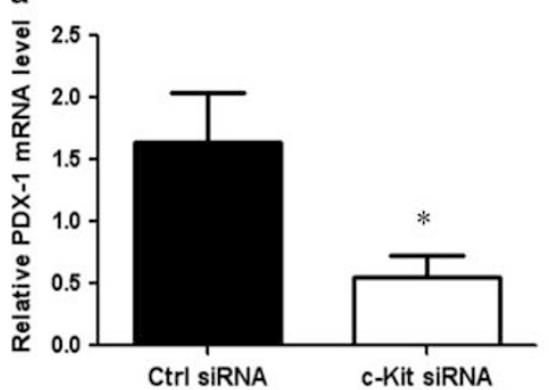

b

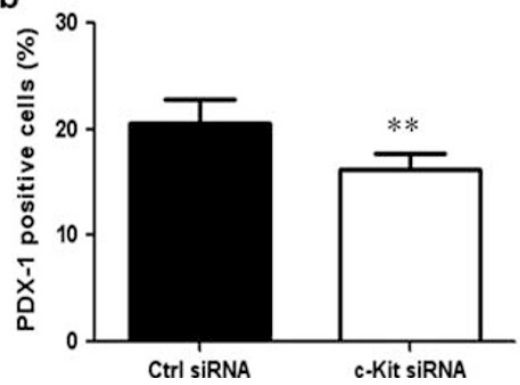

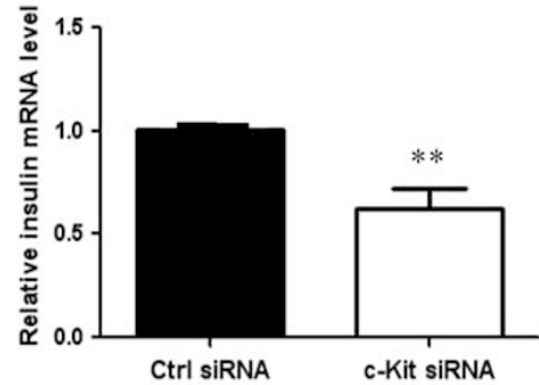

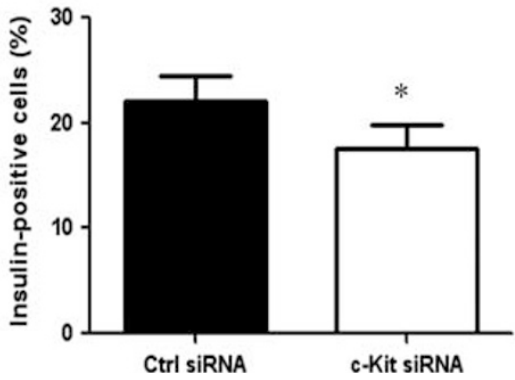

Figure 8 Effect of knockdown of c-Kit on PANC-1 cell differentiation. (a) qRT-PCR analysis of PDX-1 and insulin mRNA expression in the differentiated isletlike cell clusters from PANC-1 cells after transfection with either control or $c$-Kit siRNA. Data are normalized to the $18 \mathrm{~S}$ rRNA and expressed as means \pm s.e.m. ( $n=4$ experiments per treatment group; ${ }^{*} P<0.05,{ }^{*} p<0.01$ vs control siRNA group). (b) The percentage of PDX-1 ${ }^{+}$and insulin ${ }^{+}$cells in differentiated islet-like cell clusters treated with either control or c-Kit siRNA. Data are expressed as means \pm s.e.m. $\left(n=5\right.$ experiments per treatment group; ${ }^{\star} P<0.05$, ${ }^{*} P<0.01$ vs control siRNA group).

receptor in human fetal pancreatic cells is a crucial regulatory step during pancreatic endocrine neogenesis and for islet cell survival. ${ }^{9,11}$

In summary, our study showed that activation of the c-Kit receptor by SCF promotes PANC-1 cell differentiation into hormone-expressing endocrine cells, accompanied by increased proliferation and decreased apoptosis. These data suggest that manipulation of factors in the c-Kit/SCF signaling pathway represents a useful tool for the development of more effective cell-based therapies for diabetes treatment.

Supplementary Information accompanies the paper on the Laboratory Investigation website (http://www.laboratoryinvestigation.org)

\section{ACKNOWLEDGEMENTS}

This work was supported by grants from the Canadian Institute of Health Research (CIHR MOP89800). Dr Wang is supported by a New Investigator Award from CIHR. We are grateful to Ms M Liu for the technical assistance. We are also grateful to Dr Cindy G Goodyer (Research Institute of Montreal Children's Hospital, McGill University) for her critical comments on the paper.

\section{DISCLOSURE/CONFLICT OF INTEREST}

The authors declare no conflict of interest.

1. Sachs DH, Bonner-Weir S. New islets from old. Nat Med 2000;6:250-251.

2. Zwillich T. Diabetes research. Islet transplants not yet ready for prime time. Science 2000;289:531-533.

3. Maria-Engler SS, Mares-Guia M, Correa ML, et al. Microencapsulation and tissue engineering as an alternative treatment of diabetes. Braz J Med Biol Res 2001;34:691-697.
4. Bretzel RG, Eckhard M, Brendel MD. Pancreatic islet and stem cell transplantation: new strategies in cell therapy of diabetes mellitus. Panminerva Med 2004;46:25-42.

5. Lechner A, Habener JF. Stem/progenitor cells derived from adult tissues: potential for the treatment of diabetes mellitus. Am J Physiol Endocrinol Metab 2003;284:E259-E266.

6. Linnekin D. Early signaling pathways activated by c-Kit in hematopoietic cells. Int J Biochem Cell Biol 1999;31:1053-1074.

7. Oberg-Welsh $C$, Welsh $M$. Effects of certain growth factors on in vitro maturation of rat fetal islet-like structures. Pancreas 1996;12:334-339.

8. Rachdi L, El Ghazi L, Bernex F, et al. Expression of the receptor tyrosine kinase KIT in mature beta-cells and in the pancreas in development. Diabetes 2001;50:2021-2028.

9. Li J, Quirt J, Do HQ, et al. Expression of c-Kit receptor tyrosine kinase and effect on beta-cell development in the human fetal pancreas. Am J Physiol Endocrinol Metab 2007;293:E475-E483.

10. Yashpal NK, Li J, Wang R. Characterization of c-Kit and nestin expression during islet cell development in the prenatal and postnatal rat pancreas. Dev Dyn 2004;229:813-825.

11. Li J, Goodyer CG, Fellows F, et al. Stem cell factor/c-Kit interactions regulate human islet-epithelial cluster proliferation and differentiation. Int J Biochem Cell Biol 2006;38:961-972.

12. Wang R, Li J, Yashpal N. Phenotypic analysis of c-Kit expression in epithelial monolayers derived from postnatal rat pancreatic islets. J Endocrinol 2004;182:113-122.

13. Krishnamurthy M, Ayazi F, Li J, et al. c-Kit in early onset of diabetes: a morphological and functional analysis of pancreatic beta-cells in

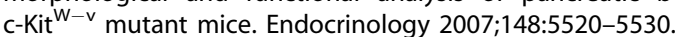

14. Lieber M, Mazzetta J, Nelson-Rees W, et al. Establishment of a continuous tumor-cell line (panc-1) from a human carcinoma of the exocrine pancreas. Int J Cancer 1975;15:741-747.

15. Githens S. The pancreatic duct cell: proliferative capabilities, specific characteristics, metaplasia, isolation, and culture. J Pediatr Gastroenterol Nutr 1988;7:486-506.

16. Hardikar AA, Marcus-Samuels B, Geras-Raaka E, et al. Human pancreatic precursor cells secrete FGF2 to stimulate clustering into hormoneexpressing islet-like cell aggregates. Proc Natl Acad Sci USA 2003;100:7117-7122. 
17. Graham FL, Smiley J, Russell WC, et al. Characteristics of a human cell line transformed by DNA from human adenovirus type 5. J Gen Virol 1977;36:59-72.

18. Mashima $\mathrm{H}$, Ohnishi $\mathrm{H}$, Wakabayashi $\mathrm{K}$, et al. Betacellulin and activin $\mathrm{A}$ coordinately convert amylase-secreting pancreatic AR42J cells into insulin-secreting cells. J Clin Invest 1996;97:1647-1654.

19. Mashima $\mathrm{H}$, Shibata $\mathrm{H}$, Mine $\mathrm{T}$, et al. Formation of insulin-producing cells from pancreatic acinar AR42J cells by hepatocyte growth factor. Endocrinology 1996;137:3969-3976.

20. Zhou J, Wang X, Pineyro MA, et al. Glucagon-like peptide 1 and exendin-4 convert pancreatic AR42J cells into glucagon- and insulinproducing cells. Diabetes 1999;48:2358-2366

21. Wang RN, Rosenberg L. Maintenance of beta-cell function and survival following islet isolation requires re-establishment of the islet-matrix relationship. J Endocrinol 1999;163:181-190.

22. Lumelsky $\mathrm{N}$, Blondel $\mathrm{O}$, Laeng $\mathrm{P}$, et al. Differentiation of embryonic stem cells to insulin-secreting structures similar to pancreatic islets. Science 2001;292:1389-1394.

23. Nichols J, Zevnik B, Anastassiadis K, et al. Formation of pluripotent stem cells in the mammalian embryo depends on the POU transcription factor Oct4. Cell 1998;95:379-391.

24. Wei C, Geras-Raaka E, Marcus-Samuels B, et al. Trypsin and thrombin accelerate aggregation of human endocrine pancreas precursor cells. J Cell Physiol 2006;206:322-328.

25. Misiti S, Anastasi E, Sciacchitano S, et al. 3,5,3'-Triiodo-L-thyronine enhances the differentiation of a human pancreatic duct cell line (hPANC-1) towards a beta-cell-like phenotype. J Cell Physiol 2005;204:286-296.

26. Habener JF, Kemp DM, Thomas MK. Minireview: transcriptional regulation in pancreatic development. Endocrinology 2005;146:1025-1034.

27. Lyttle BM, Li J, Krishnamurthy $M$, et al. Transcription factor expression in the developing human fetal endocrine pancreas. Diabetologia 2008:51:1169-1180.

28. Sander M, Neubuser A, Kalamaras J, et al. Genetic analysis reveals that PAX6 is required for normal transcription of pancreatic hormone genes and islet development. Genes Dev 1997;11:1662-1673.
29. St Onge L, Sosa-Pineda B, Chowdhury $\mathrm{K}$, et al. Pax6 is required for differentiation of glucagon-producing alpha-cells in mouse pancreas. Nature 1997;387:406-409.

30. Broudy VC, Morgan DA, Lin N, et al. Stem cell factor influences the proliferation and erythroid differentiation of the MB-02 human erythroleukemia cell line by binding to a high-affinity c-kit receptor. Blood 1993;82:436-444.

31. Konig A, Corbacioglu S, Ballmaier $M$, et al. Downregulation of c-kit expression in human endothelial cells by inflammatory stimuli. Blood 1997:90:148-155.

32. Ashman LK. The biology of stem cell factor and its receptor C-kit. Int J Biochem Cell Biol 1999;31:1037-1051.

33. Yasuda A, Sawai H, Takahashi $H$, et al. The stem cell factor/c-kit receptor pathway enhances proliferation and invasion of pancreatic cancer cells. Mol Cancer 2006;5:46.

34. Huang EJ, Nocka KH, Buck J, et al. Differential expression and processing of two cell associated forms of the kit-ligand: $\mathrm{KL}-1$ and KL-2. Mol Biol Cell 1992;3:349-362.

35. Dolci S, Williams DE, Ernst MK, et al. Requirement for mast cell growth factor for primordial germ cell survival in culture. Nature 1991;352:809-811.

36. Godin I, Deed R, Cooke J, et al. Effects of the steel gene product on mouse primordial germ cells in culture. Nature 1991;352:807-809.

37. Metcalf D, Nicola NA. Direct proliferative actions of stem cell factor on murine bone marrow cells in vitro: effects of combination with colony-stimulating factors. Proc Natl Acad Sci USA 1991;88: 6239-6243.

38. Munugalavadla V, Kapur R. Role of c-Kit and erythropoietin receptor in erythropoiesis. Crit Rev Oncol Hematol 2005;54:63-75.

39. Kambe N, Miyachi Y. A possible mechanism of mast cell proliferation in mastocytosis. J Dermatol 2002;29:1-9.

40. Ren X, Hogaboam C, Carpenter A, et al. Stem cell factor restores hepatocyte proliferation in IL-6 knockout mice following $70 \%$ hepatectomy. J Clin Invest 2003;112:1407-1418.

41. Hassan HT, Zander A. Stem cell factor as a survival and growth factor in human normal and malignant hematopoiesis. Acta Haematol 1996;95:257-262. 